\title{
RIQUEZA E DISTRIBUIÇÃO DE MACRÓFITAS AQUÁTICAS NO RIO GUARIBAS, PICOS, PIAUÍ
}

\author{
Welinton Gustavo Moreira de Sousa ${ }^{1}$ (D), Edson Lourenço da Silva ${ }^{3}$ (D), Ana Carolina Landim Pacheco ${ }^{1}$ (D), \\ Tamaris Gimenez Pinheiro ${ }^{2}$ (D), Maria Carolina de Abreu ${ }^{1}$ (D) \\ ${ }^{1}$ Curso de Licenciatura em Ciências Biológicas, Universidade Federal do Piauí, campus Senador Helvídio Nunes de \\ Barros. Rua Cícero Duarte, 905. Junco. 64607-670. Picos - PI. \\ ${ }^{2}$ Curso de Licenciatura em Educação do Campo, Universidade Federal do Piauí, campus Senador Helvídio Nunes \\ de Barros. Rua Cícero Duarte, 905. Junco. 64607-670. Picos - PI. \\ ${ }^{3}$ Instituto Federal de Educação, Ciência e Tecnologia do Piauí, IFPI, campus Picos. Avenida Pedro Marques de \\ Medeiros, s/n. Pantanal. 64606-115. \\ * Autor correspondente: wgustavo99@hotmail.com
}

\begin{abstract}
RESUMO
As macrófitas aquáticas desempenham importantes funções no ambiente aquático, contribuindo para a estruturação ambiental e para o equilíbrio biológico. O presente estudo teve como objetivo conhecer como fatores ambientais interferem na riqueza biológica e na distribuição de macrófitas aquáticas no rio Guaribas, corpo d'água do semiárido fortemente alterado por ações antrópicas, município de Picos, Piauí. Para isso, amostras foram coletadas mensalmente em cinco estações no trecho urbano do referido rio, no período de setembro/2018 a fevereiro/2019. Para a amostragem dos vegetais utilizou-se um quadrante com lados de $25 \mathrm{~cm}\left(0,0625 \mathrm{~m}^{2}\right)$. O material foi conduzido para o Laboratório de Botânica da Universidade Federal do Piauí, campus de Picos, onde foi lavado, separado e identificado. Foram listadas 24 espécies distribuídas em 13 famílias botânicas, entre as quais foram mais representativas: Poaceae (5), Cyperaceae (3), Fabaceae (3), Araceae (2), Convolvulaceae (2) e Polygonaceae (2). As formas biológicas mais comuns foram as anfíbias e emergentes, anfíbias e as flutuantes livres. A maior riqueza florística ocorreu nos meses de fevereiro e setembro com 18 e 16 espécies, respectivamente. Conclui-se que a diversidade de macrófitas aquáticas do rio Guaribas sofreu influência dos fatores ambientais e antrópicos sendo favorecida pelo evento de chuvas, sucessão de espécies e eutrofização do corpo d'água.
\end{abstract}

\section{RICHNESS AND DISTRIBUTION OF AQUATIC MACROPHYTES SPECIES IN THE GUARIBAS RIVER, PICOS, PIAUÍ STATE}

\begin{abstract}
Aquatic macrophytes play important roles in the aquatic environment, contributing to environmental structuring and biological balance. The present study had as objective to know how environmental factors interfere in the biological richness and in the distribution of aquatic macrophytes in the Guaribas river, water body of the semiarid strongly altered by anthropic actions, municipality of Picos, Piauí. For that, samples were collected monthly in five stations in the urban section of the referred river, from September / 2018 to February / 2019. For the sampling of vegetables, a quadrant with sides of $25 \mathrm{~cm}(0.0625 \mathrm{~m} 2)$ was used. The material was taken to the Botany Laboratory of the Federal University of Piauí, Picos campus, where it was washed, separated and identified. 24 species were listed, distributed in 13 botanical families, among which were most representative: Poaceae (5), Cyperaceae (3), Fabaceae (3), Araceae (2), Convolvulaceae (2) and Polygonaceae (2). The most common biological forms were amphibians and emergent, amphibious and free floating. The greatest floristic richness occurred in the months of February and September with 18 and 16 species, respectively. It is concluded that the diversity of aquatic macrophytes in the Guaribas River was influenced by environmental and anthropic factors, being favored by the event of rains, succession of species and eutrophication of the water body.
\end{abstract}




\section{INTRODUÇÃO}

São consideradas aquáticas e palustres as plantas que possuem a capacidade de resistir à submersão permanente ou periódica, ao menos de seu sistema radicular, podendo assim ocupar ambientes úmidos em alguma época do ano (Amaral et al., 2008). Fazem parte deste grupo às macrófitas aquáticas que compreendem as formas macroscópicas de vegetação aquática, incluindo macroalgas, briófitas, pteridófitas adaptadas ao ambiente aquático e as angiospermas, originárias do ambiente terrestre (Araújo et al., 2012), que precisam de solo permanentemente ou temporariamente coberto por água para preencher algumas de suas funções vitais (Neiff \& Neiff, 2003).

As macrófitas são consideradas a principal fonte produtora de matéria orgânica em ambientes aquáticos (França et al., 2009) sendo a principal mantenedora da cadeia detritívora (Trindade et al., 2010). Além disso, elas oferecem proteção, alimentação e locais de nidificação para os animais vertebrados e invertebrados (Brower \& Zar, 1984), reagem às mudanças na qualidade do ambiente em que vivem (Kastratović et al., 2018), absorvem metais pesados, óleos e outros compostos melhorando substancialmente a qualidade da água (Pompêo, 2008; Sood et al., 2012). E são importantes componentes funcionais em micro-escala do habitat aquático bem como em macro-escala na estruturação de ecossistemas e nas interações tróficas em cascata (Dibble, 2005; Rocha et al., 2019) pelo aumento da complexidade espacial dos habitats (Esteves, 2011).

Nos ambientes aquáticos tropicais, em geral, as condições climáticas tendem a favorecer o crescimento desses organismos; dentre elas citam-se: as altas temperaturas médias e as intensas radiações solares. Além desses fatores, ocorrem, ainda, pressões antrópicas que podem induzir o aparecimento de condições adequadas para o desenvolvimento desses vegetais, tais como a construção de reservatórios artificiais e a eutrofização (Bianchini-Júnior, 2003).

Contudo, em ambientes como o semiárido os eventos periódicos de secas e cheias podem provocar perturbações ambientais que causam alterações nas comunidades de macrófitas aquáticas. Nos rios do semiárido brasileiro essa dinâmica hidrológica representa o grande elemento organizador da estrutura e do funcionamento destes ecossistemas (Maltchick, 1990). Nessa região, a estação chuvosa está associada principalmente a eventos irregulares, curtos, que variam em intensidade, frequência e magnitude (Barbosa et al., 2012), podendo limitar o crescimento e até mesmo a ocorrência de macrófitas aquáticas.

Assim, abordagens de cunho florísticotaxonômico são de fundamental importância para a caracterização dos sistemas aquáticos continentais, sobremaneira, no semiárido brasileiro, onde a composição de comunidades de macrófitas aquáticas ainda é escassamente conhecida (Lima et al., 2013). Portanto, conhecer a composição florística de rios dessa região é o passo inicial para a compreensão da dinâmica e estrutura de comunidades de macrófitas aquáticas nessa região do Brasil. Com isso, o objetivo do presente trabalho foi conhecer como fatores ambientais interferem na riqueza biológica e na distribuição de macrófitas aquáticas no rio Guaribas, município de Picos, semiárido piauiense, Brasil.

\section{MATERIAL E MÉTODOS}

\section{Área de estudo}

A cidade de Picos localiza-se na região sudeste

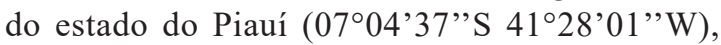
possuindo uma população estimada em 78.431 habitantes (IBGE, 2020). O clima, segundo a classificação de Köppen, é do tipo Bsh - quente e semi-árido, a estação chuvosa no verão e com precipitações que atingem uma média de $679 \mathrm{~mm}$ por ano (Aleixo et al., 2014). Picos está inserido na região semiárida (Medeiros et al., 2012), tendo como cobertura vegetal predominante a caatinga, ocorrendo pequenas manchas de cerrado à noroeste (Barbosa et al., 2007).

Dentre os principais afluentes da cidade, o rio Guaribas se destaca pela sua extensão e hidrografia, abrangendo 11 municípios (Freitas, 2002).

\section{Coleta de dados}

Foram estabelecidas cinco Estações de coleta situadas no trecho urbano do Rio Guaribas, nos bairros mais populosos do município: (1) Canto da Várzea $\left(7^{\circ} 05^{\prime} 26.1^{\prime \prime S} 41^{\circ} 28^{\prime} 35.4^{\prime} \mathrm{W}\right)$, (2) Passagem das pedras $\left(7^{\circ} 05^{\prime} 18.6^{\prime \prime} \mathrm{S} 41^{\circ} 28^{\prime} 00.6^{\prime \prime} \mathrm{W}\right)$, (3) Centro $\left(7^{\circ} 05^{\prime} 07.3^{\prime \prime S} 41^{\circ} 27^{\prime} 50.1^{\prime \prime} \mathrm{W}\right)$, (4) Boa Sorte $\left(7^{\circ} 04^{\prime} 37.4^{\prime \prime} \mathrm{S} 41^{\circ} 27^{\prime} 56.5^{\prime \prime} \mathrm{W}\right)$ e (5) Ipueiras (704'33.6”'S 41 ${ }^{\circ} 27^{\prime} 49.2^{\prime \prime} \mathrm{W}$ ) (Figura 1), respeitando a distância mínima de $100 \mathrm{~m}$ entre elas.

O percurso do rio nesses bairros é o mais afetado pela ação humana no município, contudo algumas características as diferenciam. A Estação 1 situa-se numa área cercada por árvores de grande e médio porte. O leito era constituído por regiões 


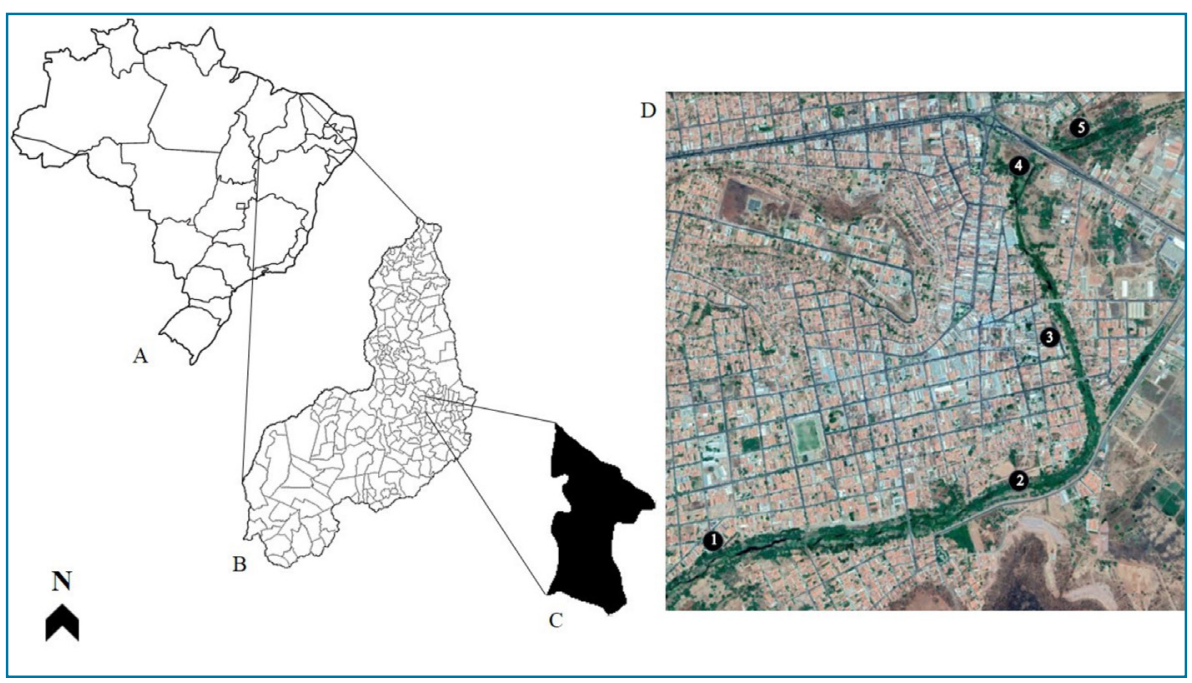

Figura 1. Localização do rio Guaribas em Picos, PI, Nordeste do Brasil, com 5 estações de coleta. Estação 1 - Canto da Várzea; Estação 2 - Passagem das Pedras; Estação 3 - Centro; Estação 4 - Boa Sorte; Estação 5 - Ipueiras. A. Mapa do Brasil - fonte: Infoescola; B. Mapa do Piauí - fonte - Wikipedia; C. Mapa de Picos - adaptado de Barbosa et al. (2007); D. Área do estudo - fonte: Google Earth.

largas e estreitas, geralmente com pouco fluxo. Apenas nesta Estação foi observada a presença de gado. A Estação 2 não apresentou tantas árvores quanto a Estação 1, sendo a vegetação arbustiva e herbácea mais comum. Nessa Estação o leito do rio se apresentou mais largo com correnteza maior do que nas demais Estações. Além disso, na Estação 2 o derramamento de esgoto foi bastante pronunciado. A Estação 3 por se situar numa região bastante habitada foi possível observar grande despejo de esgoto doméstico e a vegetação predominante foi a herbácea. As Estações 4 e 5 localizam-se numa região pouco habitada, com uma mata ciliar semelhante a da Estação 1. Com relação ao corpo d'água, tanto a Estação 4 quanto a 5 possuíam leito largo porém com baixo fluxo de água. Essas duas Estações se diferenciaram das demais pois a margem do rio Guaribas nesses locais é mais ampla, principalmente na Estação 4.

As coletas de macrófitas aquáticas ocorreram no período de seca (setembro, outubro e novembro de 2018) e período chuvoso (dezembro de 2018 e janeiro e fevereiro de 2019). Em cada Estação de coleta, foram retiradas três amostras com $5 \mathrm{~m}$ de distância entre elas.

Para a amostragem utilizou-se um quadrante de $25 \mathrm{~cm}$ de lado $\left(0,0625 \mathrm{~m}^{2}\right)$ o qual era lançado no corpo d'água à $1 \mathrm{~m}$ de distância da margem e todas as plantas contidas nele eram retiradas e armazenadas em sacos de plástico identificados. As amostras foram conduzidas para o Laboratório de Botânica da Universidade Federal do Piauí, campus de Picos, onde foram lavadas, separadas e identificadas. Além disso, foram feitas capturas de imagem para registro fotográfico do material e confirmação da identificação por meio de consultas à bibliografia especializada e especialistas. Todas as plantas coletadas foram classificadas quanto a sua forma de vida: flutuantes livres, flutuantes fixas, submersas livres, submersas fixas, emergentes e anfíbias (Irgang \& Gastal-Jr. 1996).

A cada coleta e para cada Estação foi realizada o registro das características do ambiente como: tipo e extensão da mata ciliar, erosão e assoreamento das margens, alteração antrópica, presença de dejetos, presença de animais e caracterização do fluxo da água para auxiliar na interpretação dos resultados. Dados de precipitação foram obtidos do site do Instituto Nacional de Meteorologia (INMET) disponível no site http://sisdagro.inmet.gov.br/sisdagro/app/ monitoramento/bhs.

A riqueza das macrófitas e de forma biológicas foi calculada considerando o número de espécies vegetais e de forma biológicas que ocorreram por mês e Estação de coleta. Já a frequência de ocorrência foi calculada considerando a presença de cada espécie de macrófita em cada Estação e mês de amostragem. 


\section{RESULTADOS}

$\mathrm{Na}$ área de estudo foram identificadas 24 espécies de macrófitas distribuídas em 13 famílias, sendo Poaceae $(\mathrm{n}=5 ; 20,8 \%)$, Cyperaceae $(\mathrm{n}$ $=3 ; 12,5 \%)$, Fabaceae $(\mathrm{n}=3 ; 12,5 \%)$, Araceae $(\mathrm{n}=2 ; 8,3 \%)$, Convolvulaceae $(\mathrm{n}=2 ; 8,3 \%)$ e Polygonaceae $(\mathrm{n}=2 ; 8,3 \%)$ as com maior riqueza de espécies $(70,7 \%)$, enquanto as demais famílias foram caracterizadas por uma única espécie cada (29,3\%) (Figura 2). Três espécies não tiveram sua identificação confirmada sendo denominadas respectivamente como Convolvulaceae 1, Fabaceae 1 e Poaceae 1 (Figura 2).
As formas biológicas mais representativas foram as anfíbias e emergentes $(\mathrm{n}=10)$, anfíbias $(n=7)$ e as flutuantes livres $(n=7)$ (Tabela 1). Não foram coletadas macrófitas submersas livres e fixas. As espécies Hydrocotyle bonariensis Lam., Ipomoea asarifolia (Desr.) Roem. \& Schult., Schoenoplectus sp., Ludwigia helminthorrhiza (Mart.) H. Hara, Hymenachne amplexicaulis (Rudge) Nees, Paspalidium paludivagum Hitchc. \& Chase) Parodi, Paspalum morichalense Davidse et al., Paspalum sp., Poaceae 1, Polygonum ferrugineum Wedd. e Polygonum punctatum Elliott apresentaram dois modos de vida distintos (Tabela 1).

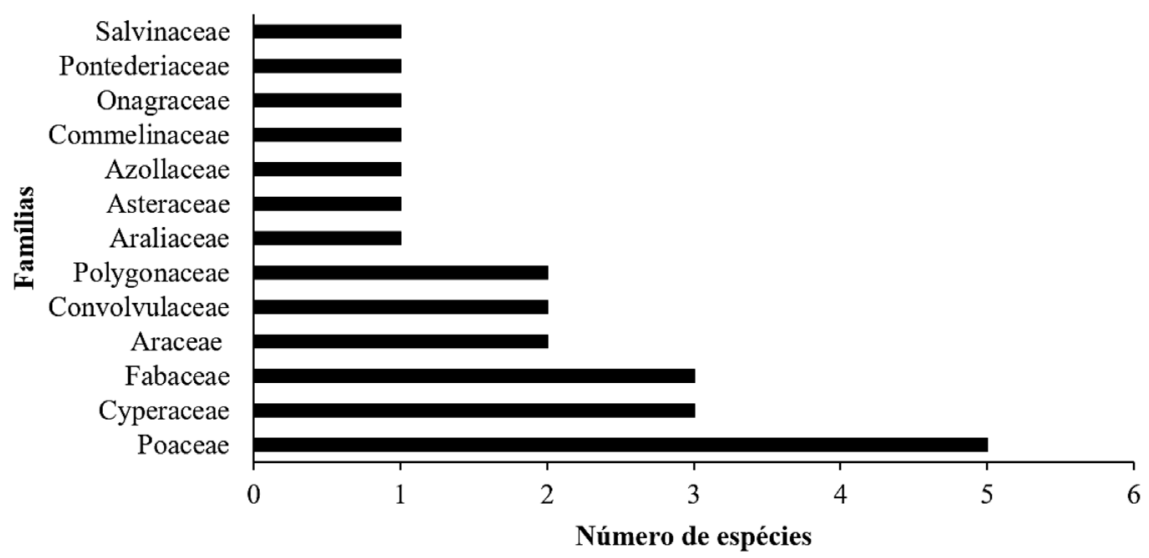

Figura 2. Número de espécies de macrófitas aquáticas distribuídas por família amostradas no trecho urbano do rio Guaribas, Piauí, Nordeste do Brasil.

Tabela 1. Lista de ocorrência mensal das espécies de macrófitas aquáticas amostradas no trecho urbano do rio Guaribas, Picos, Piauí, Nordeste do Brasil e seus modos de vida. AN: Anfíbia; E: Emergente; FL: Flutuante Livre; FF: Flutuante Fixa; 1: Presente; 0: Ausente

\begin{tabular}{|c|c|c|c|c|c|c|c|}
\hline \multirow{2}{*}{ Família/Espécie } & \multirow{2}{*}{ Modo de vida } & \multicolumn{3}{|c|}{2018} & \multicolumn{3}{|c|}{2019} \\
\hline & & Set & Out & Nov & Dez & Jan & Fev \\
\hline \multicolumn{8}{|l|}{ ARACEAE } \\
\hline Pistia stratiotes $\mathrm{L}$. & FL & 1 & 1 & 1 & 0 & 1 & 0 \\
\hline Wolffia sp. & $\mathrm{FL}$ & 1 & 1 & 1 & 0 & 1 & 0 \\
\hline \multicolumn{8}{|l|}{ ARALIACEAE } \\
\hline Hydrocotyle bonariensis Lam. & AN; E & 1 & 1 & 1 & 0 & 0 & 1 \\
\hline \multicolumn{8}{|l|}{ ASTERACEAE } \\
\hline Eclipta prostata (L.) L. & AN & 1 & 0 & 0 & 0 & 0 & 1 \\
\hline \multicolumn{8}{|l|}{ AZOLLACEAE } \\
\hline Azolla filiculoides Lam. & FL & 0 & 0 & 0 & 0 & 0 & 1 \\
\hline \multicolumn{8}{|l|}{ CONVOLVULACEAE } \\
\hline Convolvulaceae 1 & AN & 0 & 0 & 0 & 0 & 0 & 1 \\
\hline Ipomoea asarifolia (Desr.) Roem. \& Schult. & AN; E & 1 & 1 & 0 & 0 & 0 & 1 \\
\hline
\end{tabular}




\begin{tabular}{|c|c|c|c|c|c|c|c|}
\hline \multirow{2}{*}{ Família/Espécie } & \multirow{2}{*}{ Modo de vida } & \multicolumn{3}{|c|}{2018} & \multicolumn{3}{|c|}{2019} \\
\hline & & Set & Out & Nov & Dez & Jan & Fev \\
\hline
\end{tabular}

\section{COMMELINACEAE}

Commelina erecta $\mathrm{L}$.

CYPERACEAE

Cyperus digitatus Roxb.

Cyperus odoratus $\mathrm{L}$.

Schoenoplectus sp.

FABACEAE

Fabaceae 1

Neptunia plena (L.) Benth.

Senna alata (L.) Roxb.

ONAGRACEAE

Ludwigia helminthorrhiza (Mart.) H. Hara

POACEAE

Hymenachne amplexicaulis (Rudge) Nees

Paspalidium paludivagum (Hitchc. \& Chase) Parodi

Paspalum morichalense Davidse et al.

Paspalum sp.

Poaceae 1

POLYGONACEAE

Polygonum ferrugineum Wedd.

Polygonum punctatum Elliott

PONTEDERIACEAE

Eichhornia crassipes (Mart.) Solms

\section{SALVINACEAE}

Salvinia auriculata Aubl.

A precipitação no município de Picos teve uma amplitude mensal mínima de $0,03 \mathrm{~mm}$ (setembro de 2018) à máxima de 3,90 mm (fevereiro de 2019), evidenciando dois períodos climáticos distintos, um seco e outro chuvoso. O período seco compreendendo os meses de setembro, outubro e novembro de 2018 no qual foi registrado uma pluviometria de $0,37 \mathrm{~mm}$ e outro chuvoso entre dezembro de 2018 e fevereiro de 2019 com pluviometria de $5,16 \mathrm{~mm}$.

No período seco, obteve-se maior riqueza em setembro de 2018 ( $n=16$ espécies), enquanto no período chuvoso, fevereiro de 2019 foi o mês em que houve o registro da maior riqueza biológica ( $\mathrm{n}$ =18) (Figura 3). Com o advento das chuvas, houve uma redução na riqueza da flora aquática com o menor valor registrado para dezembro de 2018 ( $n=$

$\begin{array}{ccccccc}\text { AN } & 1 & 0 & 0 & 0 & 0 & 1 \\ \text { AN } & 0 & 0 & 0 & 0 & 0 & 1 \\ \text { AN } & 1 & 0 & 0 & 0 & 0 & 0 \\ \text { AN; E } & 1 & 1 & 1 & 0 & 0 & 0 \\ & & & & & & \\ \text { AN } & 0 & 0 & 0 & 0 & 0 & 1 \\ \text { FL } & 1 & 0 & 0 & 0 & 0 & 0 \\ \text { AN } & 0 & 0 & 0 & 0 & 0 & 1 \\ & & & & & & \\ \text { AN; FL } & 1 & 1 & 1 & 0 & 0 & 1 \\ & & & & & & \\ \text { AN; E } & 1 & 0 & 0 & 0 & 1 & 1 \\ \text { AN; E } & 0 & 0 & 1 & 0 & 0 & 1 \\ \text { AN; E } & 1 & 1 & 0 & 0 & 0 & 1 \\ \text { AN; E } & 1 & 0 & 0 & 0 & 1 & 1 \\ \text { AN; E } & 1 & 1 & 1 & 0 & 1 & 1 \\ & & & & & & \\ \text { AN; E } & 0 & 0 & 0 & 0 & 0 & 1 \\ \text { AN; E } & 0 & 0 & 0 & 0 & 0 & 1 \\ \text { FL } & 1 & 1 & 1 & 1 & 1 & 1 \\ \text { FL } & 1 & 1 & 1 & 1 & 0 & 0\end{array}$

2) (Figura 3). Todavia, a riqueza florística aumentou concomitante ao aumento da pluviosidade média no município (Figura 3).

As Estações com maior riqueza foram a 2 e 1, as quais detiveram $58,3 \%$ e $45,8 \%$, respectivamente, do total das espécies registradas (Figura 4). Por outro lado, a Estação 4 apresentou a menor riqueza (25\%) (Figura 4).

De forma geral, Eichhornia crassipes (Mart) Solms foi a espécie com maior frequência de ocorrência mensal (Figura 5), sendo comum nas Estações 2 e 3 em ambos os períodos climáticos (Tabela 2). Esse organismo atingiu o ápice de seu desenvolvimento propagativo e reprodutivo nos meses secos, sendo notada cobrindo a lâmina d'água em diversas partes do afluente, com exceção da Estação 5. 


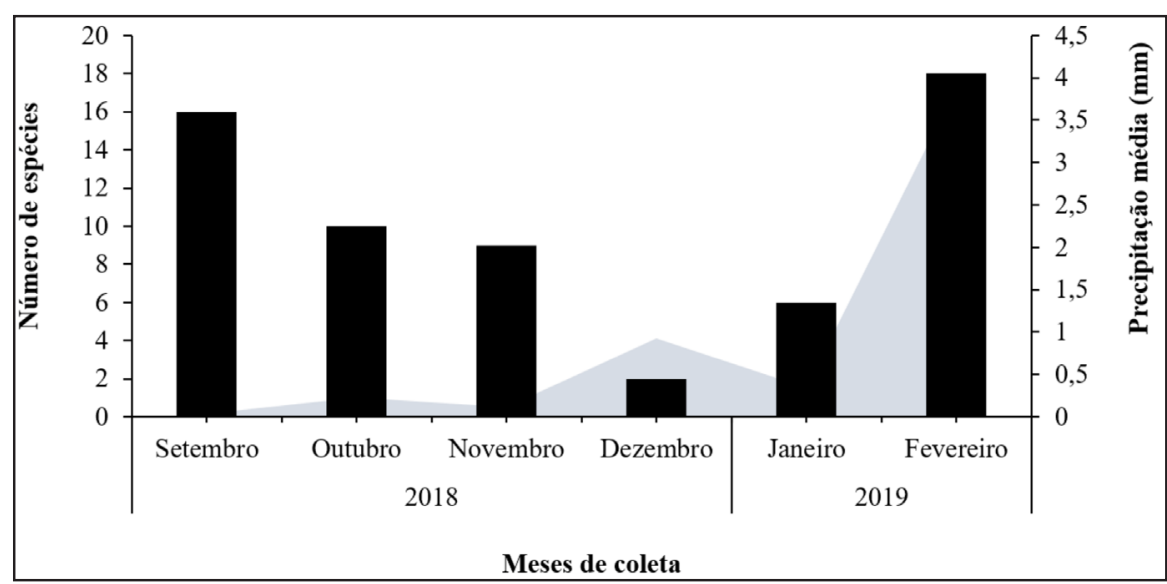

Figura 3. Diversidade de macrófitas aquáticas coletadas no trecho urbano do rio Guaribas, Piauí, Nordeste do Brasil e precipitação média mensal da região. Fonte dos dados de precipitação INMET (2020).

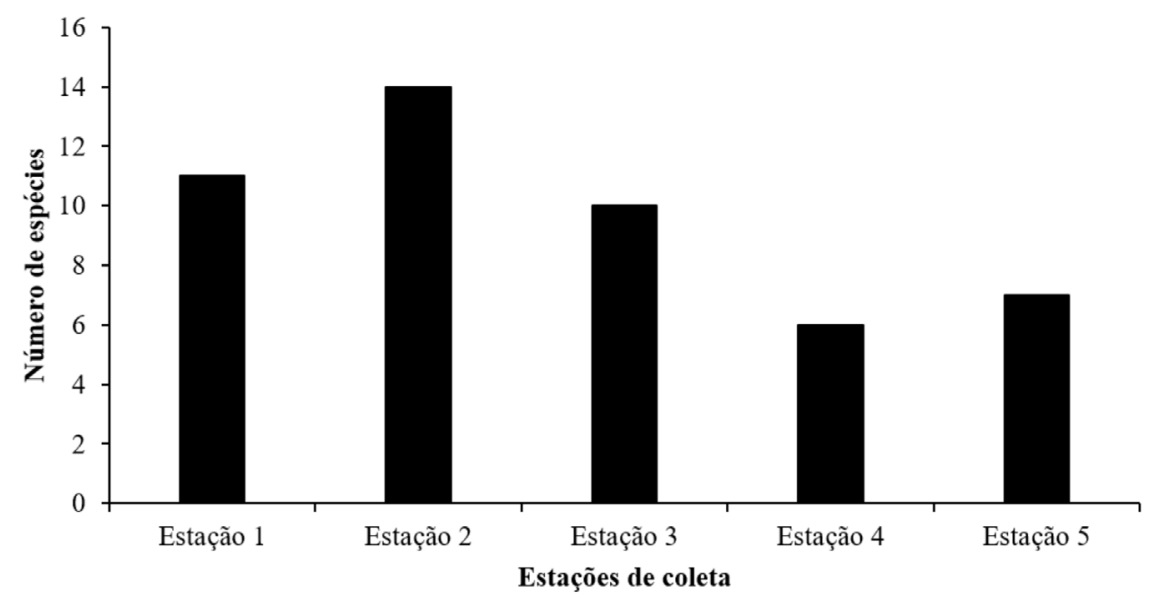

Figura 4. Diversidade de macrófitas aquáticas por estação de coleta amostradas no trecho urbano do rio Guaribas, Piauí, Nordeste do Brasil.

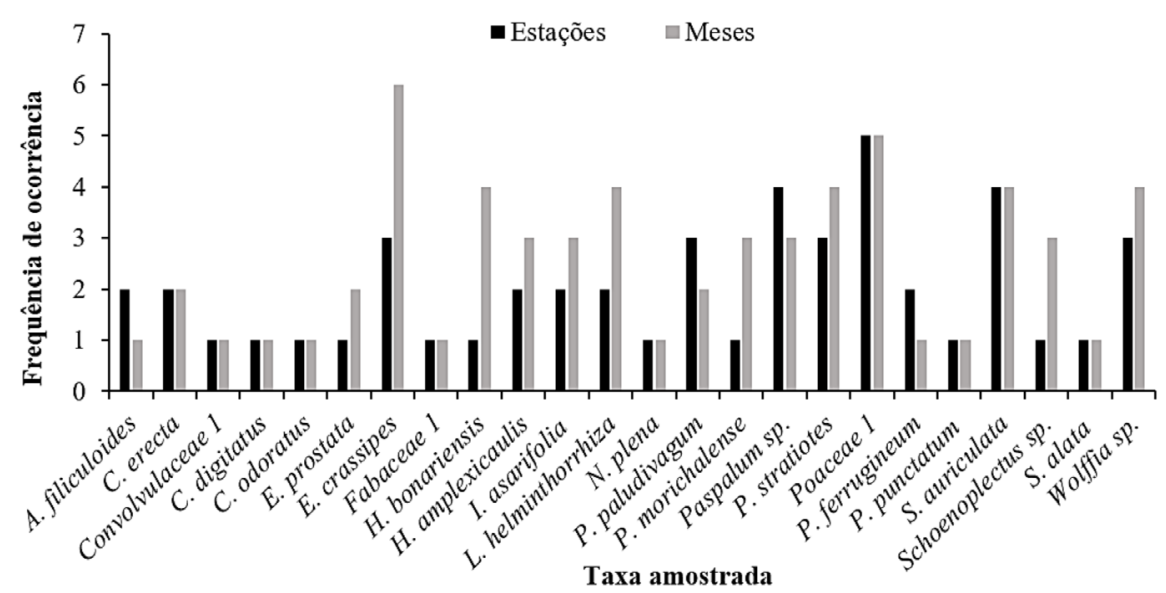

Figura 5. Frequência de ocorrência (mensal e por estação) de macrófitas aquáticas amostradas no trecho urbano do rio Guaribas, Piauí, Nordeste do Brasil. 
Representantes da família Pontederiaceae, Poaceae, Fabaceae e Araceae (E. crassipes, Poaceae 1, Salvinia auriculata e Wolffia sp., respectivamente) exibiram expressiva frequência de ocorrência no período seco (Tabela 2). Observou-se que esses vegetais tiveram preferência a regiões pouco afetadas pela correnteza, como as margens do rio ou debaixo de outras plantas. Em compensação, Paspalum sp. foi a mais predominante no período chuvoso (Tabela 2).

Diferentes características ambientais dirigidas por alterações naturais ou antropogênicas marcaram as regiões de coleta. A partir de pesquisas realizadas in loco, verificou-se que o despejo de esgoto e de resíduos sólidos foi comum a todas as estações, sendo mais pronunciado nas Estações 1, 2 e 3. A supressão da mata ciliar ou sua substituição por árvores exóticas, como a Prosopis juliflora (Sw) DC e Parkinsonia aculeata L., também foi frequente nas margens do rio. Contudo, a Estação 3 que se localizava no Centro foi a mais afetada pelo desmatamento. Em contraste, as Estações 4 e 5 encontraram-se sombreadas e, contrariando as demais Estações, passaram boa parte do período seco sem fluxo de água. Na Estação 1 houve uma acentuada presença de mamíferos herbívoros, além de instalações de currais e chiqueiros nas margens do rio.

Tabela 2. Lista de ocorrência das espécies de macrófitas aquáticas por estação de coleta do trecho urbano do rio Guaribas, Picos, Piauí, Nordeste do Brasil. 1: Presente; 0: Ausente.

\begin{tabular}{|c|c|c|c|c|c|c|c|c|c|c|}
\hline \multirow{3}{*}{ Família/ Espécies } & \multicolumn{10}{|c|}{ Ocorrência nas estações } \\
\hline & \multicolumn{5}{|c|}{$\begin{array}{c}\text { Período de seca } \\
\text { (setembro a novembro } \\
\text { de 2018) }\end{array}$} & \multicolumn{5}{|c|}{$\begin{array}{l}\text { Período de chuva } \\
\text { (dezembro de } 2018 \text { a } \\
\text { fevereiro de 2019) }\end{array}$} \\
\hline & 1 & 2 & 3 & 4 & 5 & 1 & 2 & 3 & 4 & 5 \\
\hline \multicolumn{11}{|l|}{ ARACEAE } \\
\hline Pistia stratiotes L. & 1 & 1 & 0 & 0 & 0 & 0 & 1 & 0 & 1 & 0 \\
\hline Wolffia sp. & 1 & 1 & 1 & 0 & 0 & 0 & 1 & 0 & 0 & 0 \\
\hline \multicolumn{11}{|l|}{ ARALIACEAE } \\
\hline Hydrocotyle bonariensis Lam. & 0 & 0 & 0 & 0 & 1 & 0 & 0 & 0 & 0 & 1 \\
\hline \multicolumn{11}{|l|}{ ASTERACEAE } \\
\hline Eclipta prostata (L.) L. & 0 & 1 & 1 & 0 & 0 & 0 & 1 & 0 & 0 & 0 \\
\hline \multicolumn{11}{|l|}{ AZOLLACEAE } \\
\hline Azolla filiculoides Lam. & 0 & 0 & 0 & 0 & 0 & 1 & 1 & 0 & 0 & 0 \\
\hline \multicolumn{11}{|l|}{ CONVOLVULACEAE } \\
\hline Convolvulaceae 1 & 0 & 0 & 0 & 0 & 0 & 0 & 0 & 1 & 0 & 0 \\
\hline Ipomoea asarifolia (Desr.) Roem. \& Schult. & 0 & 0 & 0 & 0 & 1 & 1 & 0 & 0 & 0 & 0 \\
\hline \multicolumn{11}{|l|}{ COMMELINACEAE } \\
\hline Commelina erecta $\mathrm{L}$. & 0 & 0 & 1 & 0 & 0 & 0 & 1 & 1 & 0 & 0 \\
\hline \multicolumn{11}{|l|}{ CYPERACEAE } \\
\hline Cyperus digitatus Roxb. & 0 & 0 & 0 & 0 & 0 & 0 & 1 & 0 & 0 & 0 \\
\hline Cyperus odoratus L. & 0 & 1 & 0 & 0 & 0 & 0 & 0 & 0 & 0 & 0 \\
\hline Schoenoplectus sp. & 0 & 1 & 0 & 0 & 0 & 0 & 0 & 0 & 0 & 0 \\
\hline \multicolumn{11}{|l|}{ FABACEAE } \\
\hline Fabaceae 1 & 0 & 0 & 0 & 0 & 0 & 0 & 0 & 1 & 0 & 0 \\
\hline Neptunia plena (L.) Benth. & 0 & 0 & 0 & 1 & 0 & 0 & 0 & 0 & 0 & 0 \\
\hline Senna alata (L.) Roxb. & 0 & 0 & 0 & 0 & 0 & 0 & 0 & 1 & 0 & 0 \\
\hline
\end{tabular}




\begin{tabular}{|c|c|c|c|c|c|c|c|c|c|c|}
\hline \multirow{3}{*}{ Família/ Espécies } & \multicolumn{10}{|c|}{ Ocorrência nas estações } \\
\hline & \multicolumn{5}{|c|}{$\begin{array}{c}\text { Período de seca } \\
\text { (setembro a novembro } \\
\text { de 2018) }\end{array}$} & \multicolumn{5}{|c|}{$\begin{array}{l}\text { Período de chuva } \\
\text { (dezembro de } 2018 \text { a } \\
\text { fevereiro de 2019) }\end{array}$} \\
\hline & 1 & 2 & 3 & 4 & 5 & 1 & 2 & 3 & 4 & 5 \\
\hline \multicolumn{11}{|l|}{ ONAGRACEAE } \\
\hline Ludwigia helminthorrhiza (Mart.) H. Hara & 1 & 1 & 0 & 0 & 0 & 0 & 1 & 0 & 0 & 0 \\
\hline \multicolumn{11}{|l|}{ POACEAE } \\
\hline Hymenachne amplexicaulis (Rudge) Nees & 0 & 1 & 0 & 0 & 0 & 0 & 1 & 1 & 0 & 0 \\
\hline $\begin{array}{l}\text { Paspalidium paludivagum (Hitchc. \& Chase) Pa- } \\
\text { rodi }\end{array}$ & 0 & 0 & 0 & 1 & 0 & 1 & 0 & 1 & 0 & 0 \\
\hline Paspalum morichalense Davidse et al. & 0 & 0 & 0 & 0 & 1 & 0 & 0 & 0 & 0 & 1 \\
\hline Paspalum sp. & 0 & 0 & 0 & 1 & 0 & 1 & 1 & 0 & 1 & 1 \\
\hline Poaceae 1 & 1 & 1 & 1 & 1 & 1 & 0 & 1 & 0 & 1 & 1 \\
\hline \multicolumn{11}{|l|}{ POLYGONACEAE } \\
\hline Polygonum ferrugineum Wedd. & 0 & 0 & 0 & 0 & 0 & 0 & 1 & 0 & 0 & 1 \\
\hline Polygonum punctatum Elliott & 0 & 0 & 0 & 0 & 0 & 1 & 0 & 0 & 0 & 0 \\
\hline \multicolumn{11}{|l|}{ PONTEDERIACEAE } \\
\hline Eichhornia crassipes (Mart.) Solms & 0 & 1 & 1 & 0 & 0 & 1 & 1 & 1 & 0 & 0 \\
\hline \multicolumn{11}{|l|}{ SALVINACEAE } \\
\hline Salvinia auriculata Aubl. & 1 & 0 & 1 & 0 & 0 & 0 & 1 & 1 & 0 & 0 \\
\hline
\end{tabular}

\section{DISCUSSÃO}

Em Picos, o rio Guaribas apresenta sérios problemas ambientais, principalmente na zona urbana, em função da forma de uso e ocupação de seu leito e margens (Santos et al., 2015), os quais sofrem interferência humana, através de dejetos de hospitais, esgotos domésticos e resíduos sólidos (Costa et al., 2018). Esses problemas afetam diretamente a paisagem e interferem na qualidade das águas e em sua dinâmica natural (Sousa \& Lima, 2019). Apesar disso, a diversidade florística foi bastante representativa, superior ao obtido por Dias et al. (2017) em um reservatório no semiárido brasileiro, no qual foram levantadas 13 espécies pertencentes a 12 famílias.

A maior riqueza de Cyperaceae e Poaceae tem sido comumente catalogada em diversos estudos de cunho florístico em ambientes aquáticos no Brasil (Henry-Silva et al., 2010; Mormul et al., 2010; Pinto et al., 2010; Pivari et al., 2011; Lima et al., 2013; Torres et al., 2016; Moura-Júnior \& Cotarelli, 2019). Tanto Poaceae quanto Cyperaceae são famílias com distribuição cosmopolita, apresentando grande número de espécies. Além disso, Poaceae e
Cyperaceae possuem espécies que são estoloníferas, uma característica que as torna muito mais propensas a expandir sua distribuição e a mostrar distribuição dominantes em contraste com espécies de outras famílias, (Souza et al., 2017).

Com relação as formas biológicas das plantas aquáticas identificadas no presente estudo, os dados obtidos demonstram que embora algumas espécies apresentem um único modo de vida, a maioria tem uma plasticidade adaptativa possuindo mais de uma forma biológica, característica de um alto grau de adaptação (Rocha et al., 2007). A predominância de espécies anfíbias e emergentes é citada em diversos estudos (Pott \& Pott, 1997; França et al., 2003; Ferreira et al., 2010; Moura et al., 2010; Gomes \& Aoki, 2015; Silva \& Fontes, 2018). A alta riqueza das plantas que exibem essas duas formas biológicas está associada às adaptações para: sobrevivência em corpos d'água com baixa profundidade (Neves et al., 2006; Pinheiro \& Jardim, 2015); suportar as oscilações no nível da água (Rocha et al., 2012); e se valer da alta concentração de nutrientes disponíveis (Pereira et al., 2012).

A maior frequência das plantas anfíbias e emergentes também tem relação com a expressiva 
riqueza das famílias Fabaceae, Poaceae e Cyperaceae que predominaram na amostragem, corroborando Lima et al. (2011) que afirmaram que a representatividade dessas famílias pode estar ligada a características adaptativas, morfológicas, reprodutivas e competitivas que algumas delas possuem. Segundo Ferreira (2005), as espécies anfíbias por serem na sua maioria invasoras, apresentam maiores percentuais de ocorrência, sendo um bom indicativo do grau de perturbação e/ou eutrofização a que os ambientes estão sujeitos (Pereira et al., 2012). Do mesmo modo, espécies emergentes e anfíbias têm forte tolerância ao estresse hídrico e podem facilmente se adaptar a condições de inundações e secas (Souza et al., 2017).

$\mathrm{O}$ efeito do fenômeno de eutrofização foi observado no aumento da frequência das espécies flutuantes livres E. crassipes, S. auriculata e Wolffia sp. no período seco devido à vultuosa disponibilidade de nutrientes na água advindos da morte das plantas, dos esgotos e da retração das águas do rio Guaribas. Para o referido rio, Santos et al. (2017) também relacionaram o aparecimento das espécies E. crassipes e $S$. auriculata à intensa eutrofização do ambiente lótico. Salvinia auriculata foi vista ocorrendo principalmente na Estação 4, numa região sombreada e sem fluxo d'água, corroborando com os resultados obtidos por Santos et al. (2017), os quais verificaram a ocorrência dessa planta em locais de água parada ou com pouco deslocamento. Ademais, as espécies de Wolffia são comuns nas margens, ocorrendo em associação a outras espécies de 'Lemnaceae' assim como em meio à raízes de Pistia stratiotes, Eichhornia crassipes, Liminobium stoleniferum e Azolla filiculoides, como foi verificado por Bezerra \& França (1999) em um trabalho envolvendo a ordem Arales em uma área do semiárido baiano.

A exposição do substrato durante períodos de níveis de água extremamente baixos pode facilitar a invasão de espécies agressivas e/ou exóticas (Hudon, 1997), aumentando o impacto no ambiente e nas relações biológicas (Beyruth, 1992). Assim, a redução do nível da água no período seco causou a morte de diversas plantas, tornando o ambiente propício ao desenvolvimento de espécies invasoras e daninhas, em especial à flutuante livre E. crassipes, conforme já apontado por Lima et al. (2011).

$\mathrm{Na}$ Estação 2 a hidrodinâmica lenta e estável causada pelo derramamento de esgoto e a menor profundidade propiciaram sua colonização por ervas daninhas altamente produtivas, como E. crassipes (tabela 3), que foi vista formando grandes estandes sobre a lâmina d'água no período de estiagem. Por outro lado, a maior largura do rio nessa região, as margens pouco desenvolvidas e a forte incidência de luz solar propiciaram o povoamento da margem do leito por espécies emergentes e anfíbias. Dessa forma, a maior riqueza registrada na Estação 2 é, provavelmente, resultado de sua localização em uma região onde ocorre maior sedimentação e áreas mais rasas são formadas, proporcionado um ambiente adequado para colonização por macrófitas aquáticas de diferentes espécies e formas de vida. Outro fator seria a grande área de vegetação terrestre alagada, onde a decomposição contribui para o aumento das concentrações de nutrientes, de acordo com Lolis \& Thomaz (2011).

A eutrofização é um fator que está relacionado ao desequilíbrio das comunidades de macrófitas aquáticas. Conforme Thomaz \& Bini (1998), durante a eutrofização, diferentes grupos ecológicos de macrófitas aquáticas colonizam os ecossistemas aquáticos. No entanto, nos estágios mais avançados da eutrofização, as macrófitas submersas enraizadas geralmente entram em declínio, em virtude da redução da penetração da luz, causada pelo desenvolvimento do fitoplâncton (Neves et al., 2006) e/ou pela presença de macrófitas flutuantes (Santos \& Lolis, 2012). Essas interferências favoreceram o aumento da quantidade de matéria orgânica presente na coluna d'água, beneficiando a diminuição na transparência e na incidência de luz solar (Neves et al., 2006; Rocha et al., 2012), acarretando perda na diversidade total de macrófitas aquáticas (Esteves, 2011; Milhomens et al., 2016). Assim, a turbidez da água (Kufner et al., 2011) e os efeitos da diminuição da irradiação têm sido sugeridos como as principais razões para o desaparecimento de macrófitas submersas em águas eutróficas devido aos efeitos de sombreamento (Yuan et al., 2012).

Por outro lado, as Poaceae frequentes nas Estações 4 e 5, representam o principal componente das formações campestres em todo o mundo (Sousa \& Lorenzi, 2005). Portanto, a sua presença na Estação 5 pode ser atribuída à menor profundidade do ambiente na maior parte do período de coleta (Araújo et al., 2012). Além disso, a alta densidade de gramíneas formando ilhotes observada nas margens do rio Guaribas, nessa estação, confirma o que Beyruth (1992) atribuiu como sendo determinantes para o não ingresso de macrófitas livre flutuantes no corpo d'água.

No entanto, as enchentes foram a força motriz na distribuição das comunidades de macrófitas aquáticas do rio Guaribas, afetando tanto a riqueza 
(de espécies e formas biológicas) quanto a frequência de ocorrência dessas plantas. No caso do ambiente estudado, a chuva no mês de dezembro de 2018 serviu para dispersar no ambiente as espécies de maior frequência, limitando a ocorrência desses organismos no rio. Isso foi descrito por Pedro et al. (2006) em estudo sobre o papel do ciclo hidrológico na dinâmica de macrófitas aquáticas em rios do semiárido brasileiro, onde foi notado que a intensidade das cheias foi determinante no início da recolonização, na produtividade e na biomassa da vegetação. Segundo Mauhs et al. (2006), durante as cheias, as espécies de ocorrência esporádica ou ocasional podem ser favorecidas se estabelecendo e preenchendo novos sítios de colonização, o que foi comprovado no presente estudo. Dessa forma, pode-se afirmar que o ciclo hidrológico influencia o aumento na riqueza local, proporcionando a colonização por espécies menos competitivas, retrocedendo aos estágios iniciais de sucessão conforme apontado por Santos \& Thomaz (2005).

Entretanto, a riqueza da flora aquática cresceu à medida que a precipitação aumentou no município, sendo observado um ápice em ambos os caracteres no mês de fevereiro de 2019. O aumento no intervalo entre os distúrbios, como as enchentes e secas, podem aumentar a heterogeneidade biológica, pois há mais tempo e espaço disponível para a invasão e propagação de novas espécies (Connel, 1978). Assim, as perturbações em conjunto aos gradientes ambientais podem agir positivamente entre a conectividade e a heterogeneidade espaço-temporal, elevando a riqueza biológica local (Ward, 1998).

\section{CONCLUSÃO}

Em regiões sazonalmente influenciadas como o semiárido, quando um novo regime hídrico se estabelece ocorre mudanças drásticas na distribuição de plantas aquáticas e na composição da comunidade em função no novo padrão de estresse ambiental do rio. A riqueza de macrófitas aquáticas do rio Guaribas sofreu influência dos fatores ambientais e antrópicos sendo favorecida pelo evento de chuvas, sucessão de espécies e eutrofização do corpo d'água.

\section{AGRADECIMENTOS}

Os dados deste estudo são oriundos dos resultados parciais do ICV (Iniciação Científica Voluntária) do primeiro autor. A Universidade Federal do Piauí, campus Senador Helvídio Nunes de Barros e Instituto Federal do Piauí, campus
Picos pelo apoio logístico. A todos que compões o Laboratório de Botânica - LABOT (UFPI - CSHNB) e o Laboratório de Parasitologia, Ecologia e Doenças Negligenciadas - LAPEDONE (UFPI - CSHNB), pelo apoio e orientação.

\section{REFERÊNCIAS}

ALEIXO, D. L.; ARAÚJO, W. L.; AGRA, R. S.; MARACAJÁ, P. B.; SOUSA, M. J. O. 2014. Mapeamento da flora apícola arbórea das regiões pólos do estado do Piauí. Revista Verde de Agroecologia e Desenvolvimento Sustentável, 9(4): 262 - 270.

AMARAL, M. C. E., BITTRICH, V., FARIA, A. D., ANDERSON, L. O., AONA, L. Y. S. 2008. Guia de campo para plantas aquáticas e palustres do Estado de São Paulo. Ribeirão Preto: Holos, Editora. Série "Manuais Práticos em Biologia". 452 p.

ARAÚJO, E. S., SABINO, J. H. F., COTARELLI, V. M., FILHO, J. A. S., CAMPELO, M. J. A. 2012. Riqueza e diversidade de macrófitas aquáticas em mananciais da Caatinga. Diálogos \& Ciência, 10(32): 229-233.

BARBOSA, J. E. L., MEDEIROS, E. S. F., BRASIL, J., CORDEIRO, R. S., CRISPIM, M. C. B., SILVA, G. H. G. 2012. Sistemas aquáticos no semi-árido brasileiro: limnologia e manejo. Acta Limnologica Brasiliensia, 24 (1): 103-118.

BARBOSA, M. P; NETO, J. M. M.; FERNANDES, M. F.; SILVA, M. J. 2007. Estudo da degradação das terras - município de Picos - PI. Anais XIII Simpósio Brasileiro de Sensoriamento Remoto, Florianópolis, Brasil, 21-26 abril 2007, INPE, 4357-4363.

BEYRUTH, Z. 1992. Macrófitas aquáticas de um lago marginal ao rio Embu-mirim, São Paulo, Brasil. Revista Saúde Pública, 26(4): 272-282.

BEZERRA, M. G. \& FRANÇA, F. 1999. Arales de lagoas em uma área do semi-árido baiano. Sitientibus, 20: $45-54$.

BIANCHINI-JÚNIOR, I. 2003. Modelos de crescimento e decomposição de macrófitas aquáticas. In: Thomaz, S. M. \& Bini, L. M. (org.). Ecologia e Manejo de Macrófitas Aquáticas. Maringá: EDUEM. 85-126.

BROWER, J. E. \& ZAR, J. H. 1984. Field and laboratory methods for general ecology. $2 \mathrm{ed}$. Dubuque: W. M. C. Brow.

CONNELL, J. H. 1978. Diversity in Tropical Rain Forests and Coral Reefs. Science, New Series, 
199(4335): 1302-1310.

COSTA, F. N. L., OLIVEIRA, Y. R., SILVA, P. H., FERREIRA, P. M. P., PACHECO, A.C. L., ABREU, M. C. 2018. Avaliação da qualidade hídrica de um rio do semiárido piauiense. Journal of Environmental Analysis and Progress, 3(2): 218-225.

DIAS, A. S., CAVALCANTE, S. C., NASCIMENTO, K. J. RANGEL, A. J. 2017. ASPECTOS FLORÍSTICOS E IMPLICAÇÕES ECOLÓGICAS DAS COMUNIDADES DE MACRÓFITAS AQUÁTICAS DE RESERVATÓRIO DO SEMIÁRIDO. In: Educação ambiental: natureza, biodiversidade e sociedade / Giovanni Seabra (Organizador). Ituiutaba: Barlavento, 2017.

DIBBLE, E. D. 2005. O papel ecológico das plantas aquáticas nos corredores de biodiversidade. In: Cadernos da Biodiversidade / Diretoria de Biodiversidade e Áreas Protegidas, Instituto Ambiental do ParanÁ (org.), Curitiba. 1(1): 4-13.

ESTEVES, F. A. 2011. Fundamentos de Limnologia. 3. ed. Rio de Janeiro: Interciência.

FERREIRA, F. A. 2005. Comunidades de macrófitas aquáticas e aspectos físicos-químicos de três lagoas do Parque Estadual do Rio Doce, MG. Tese de mestrado, Universidade Federal de Viçosa, Viçosa.

FERREIRA, F. A., MORMUL1, R. P., PEDRALLI, G., POTT, V. J., \& POTT, A. 2010. Estrutura da comunidade de macrófitas aquáticas em três lagoas do Parque Estadual do Rio Doce, Minas Gerais, Brasil. Hoehnea, 37(1): 43-52.

FRANÇA, C. R. C., FREIRE, O. D., SANTOS, V. V., MOURA-JÚNIOR, A. M., SEVERI, W., MAGALHÃES, K. M. 2009. Biomassa de Macrófitas Aquáticas no Reservatório de Xingó, SE/AL. In: IX Jornada de Ensino, Pesquisa e Extensão, Recife - PE. Semana Nacional de Ciência e Tecnologia.

FRANÇA, F., MELO, E. NETO, A. G., ARAÚJO, D., BEZERRA, M., RAMOS, H, M., CASTRO, I., GOMES, D. 2003. Flora vascular de açudes de uma região do semi-árido da Bahia, Brasil. Acta Botânica Brasilica, 17(4): 549-559.

FREITAS, M. A. S. 2002. Usos Múltiplos da Água na Bacia Hidrográfica do Rio Guaribas (Estado do Piauí). In: VI Simpósio de Recursos Hídricos do Nordeste, Maceió. Anais do VI Simpósio de Recursos Hídricos do Nordeste. Porto Alegre: Editora da ABRH, v. 1.

GOMES, A. C. \& AOKI, C. 2015. Efeito da sazonalidade hídrica sobre a fitossociologia de macrófitas aquáticas em uma lagoa no Pantanal, Brasil. Rev. Biol. Neotrop. 12(1): 1-7.

HENRY-SILVA, G. G., MOURA, R. S. T., DANTAS, L. L. O. 2010. Richness and distribution of aquatic macrophytes in Brazilian semi-arid aquatic ecosystems. Acta Limnologica Brasiliensia, 22(2): 147-156.

HUDON, C. 1997. Impact of water level fluctuations on St. Lawrence River aquatic vegetation. Can. J. Fish. Aquat. Sci. 54: 2853-2865.

INSTITUTO BRASILEIRO DE GEOGRAFIA E ESTATÍSTICA (IBGE). 2020. Cidades e Estados. Disponível em: https://cidades.ibge.gov.br/brasil/pi/ picos/panorama. Acesso em: outubro de 2020.

INSTITUTO NACIONAL DE METEOROLOGIA (INMET). 2020. Chuva para o município de Picos-PI, 2018-2019. Disponível em: https://portal.inmet.gov. br/. Acesso em: 11 de outubro de 2020.

IRGANG, B.E. \& GASTAL JR., C.V.S. 1996. Macrófitas aquáticas da Planície Costeira do RS. Universidade Federal do Rio Grande do Sul, Porto Alegre. 289p.

KASTRATOVIĆ, V., BIGOVIĆ, M., JAĆIMOVIĆ, Ž., KOSOVIĆ, M., ĐUROVIĆ, D., \& KRIVOKAPIĆ, S. 2018. Levels and distribution of cobalt and nickel in the aquatic macrophytes found in Skadar Lake, Montenegro. Environmental Science and Pollution Research, 25(27): 26823-26830.

LIMA, E. A., MACHADO-FILHO, H. O. \& MELO, J. I. M. 2013. Angiospermas aquáticas da Área de Proteção Ambiental (APA) do Cariri, Paraíba, Brasil. Rodriguésia, 64(4): 667-683.

LIMA, L. F., SILVA, S. S. L., MOURA-JÚNIOR, E. G., ZICKEL, C. S. 2011. Composição florística e chave de identificação das macrófitas aquáticas ocorrentes em reservatórios do estado de Pernambuco. Rodriguésia, 62(4): 771-783.

LOLIS, S. F. \& THOMAZ, S. M. 2011. Monitoramento da composição específica dacomunidade de macrófitas aquáticas noreservatório Luis Eduardo Magalhães. Planta daninha, 29(2): 1-10.

MALTCHIK, L. 1990. Ecologia de rios intermitentes tropicais. In: M.L.M. Pompêo (ed). Perspectivas da limnologia no Brasil. São Luís, União. p. 77-89.

MAUHS, J., MARCHIORETTO, M. S., BUDKE, J. C. 2006. Riqueza e biomassa de macrófitas aquáticas em uma área úmida na planície costeira do Rio 
Grande do Sul, Brasil. Pesquisas Botânicas, 57(1): 289-302.

MEDEIROS, R. M., TAVARES, A. L., KASSAR, C. B., SILVA, J. A. S., SILVA, V. P. R. 2012. Metodologias de cálculo da temperatura média diária do ar: aplicação para os municípios de Parnaíba, Picos e Gilbués, PI. Revista Brasileira de Agricultura Irrigada, 6(4): 283 - 295.

MILHOMENS, L. B. S., MOURA-JÚNIOR, E. G., RODRIGUES, R.G. 2016. Composição, riqueza e biomassa de macrófitas aquáticas no trecho do rio São Francisco onde será captada água para o Eixo Norte do PISF. I Simpósio da Bacia Hidrográfica do Rio São Francisco, 1-5.

MORMUL, R. P., FERREIRA, F. A., MICHELAN, T. S., CARVALHO, P., SILVEIRA, M. J., THOMAZ, S. M. 2010. Aquatic macrophytes in the large, subtropical Itaipu Reservoir, Brazil. Revista de Biologia Tropical, 58(4): 1437-1452.

MOURA, A. N., ARAUJO, E. L., BITTENCOURTOLIVEIRA, M. C., PIMENTEL, R. M. M., ALBUQUERQUE, U. P. 2010. Reservatórios do nordeste do Brasil: biodiversidade, Ecologia e Manejo, Bauru, São Paulo: Canal6.

MOURA-JÚNIOR, E. G. \& COTARELLI, V. M. 2019. An update on the knowledge of aquatic macrophytes in Northeast Brazil. Rodriguésia, 70: $1-11$.

NEIFF, J. J. \& NEIFF, A. S. G. P. 2003. Connectivity processes as a basis for the management of aquatic plants. In: Thomaz, S. M. \& Bini, L. M. (org.). Ecologia e manejo de macrófitas aquáticas. Maringá, EDUEM. p. 39-58.

NEVES, E.L., LEITE, K. R. B., FRANÇA, F., MELO, E. 2006. Plantas aquáticas vasculares em uma lagoa de planície costeira no município de Candeias, Bahia, Brasil. Sitientibus, Série Ciências Biológicas, 6(1): 24- 29.

PEDRO, F., MALTCHICK, L. \& BIANCHINIJÚNIOR, I. 2006. Hydrologic cycle and dynamics of aquatic macrophytes in two intermittent rivers of the semi-arid region of Brazil. Braz. J. Biol., 66(2B): 575-585.

PEREIRA, SA, TRINDADE, CRT, ALBERTONI, EF E PALMA-SILVA, C. 2012. Aquatic macrophytes as indicators of water quality in subtropical shallow lakes, Southern Brazil. Acta Limnologica Brasiliensia, 24 (1): 52-63.
PINHEIRO, M. N. M. \& JARDIM, M. A. G. 2015. Composição florística e formas biológicas de macrófitas aquáticas em lagos da Amazônia Ocidental, Roraima, Brasil. Biota Amazônia, 5(3):2327.

PINTO, M. P., BINI, L. M., SOUZA, D. C. 2010. In: Cadernos da Biodiversidade / Diretoria de Biodiversidade e Areas Protegidas, Instituto Ambiental do Parana (org.), Curitiba. 1(1): 25-34.

PIVARI, M. O., OLIVEIRA, V. B., COSTA, F. M., FERREIRA, R. M., SALINO, A. 2011. Macrófitas aquáticas do sistema lacustre do Vale do Rio Doce, Minas Gerais, Brasil. Rodriguésia, 62(4): 759-770.

POMPEO, M. 2008. Monitoramento e manejo de macrófitas aquáticas. Ecol. Bras., 12(3): 406-424.

POTT, V. J. \& POTT, A. 1997. CHECKLISTDAS MACRÓFITAS AQUÁTICAS DO PANTANAL, BRASIL. Acta bot. bras., 11(2): 215-227.

ROCHA, C. G., RESENDE, U. M., LUGNANI, J. S. 2007. Diversidade de macrófitas em Ambientes aquáticos do IPPAN na Fazenda Santa Emília, Aquidauana, MS. Revista Brasileira de Biociências, 5(2): 456-458.

ROCHA, C. M. C., ALVES, A. E., CARDOSO, A. S., CUNHA, M. C. C. 2012. Macrófitas Aquáticas como Parâmetro no Monitoramento Ambiental da Qualidade da Água. Revista Brasileira de Geografia Física, 4: 970-983

ROCHA, C. M. C., LIMA, D., CUNHA, M. C. C., ALMEIDA, J. S. 2019. Aquatic macrophytes and trophic interactions: A scientometric analyses and research perspectives. Braz. J. Biol, 79: 617-624.

SANTOS, A. M. \& THOMAZ, S. M. 2005. Diversidade de espécies de macrófitas aquáticas em lagoas de uma planície de inundação tropical: o papel de conectividade e do nível da água. In: Cadernos da Biodiversidade / Diretoria de Biodiversidade e Areas Protegidas, Instituto Ambiental do Parana (org.), Curitiba. 1(1): 4-13. 17-24.

SANTOS, M. R. B., ABREU, M. C., PERON, A. P., DEUS, M. S. M. 2017. Macrófitas aquáticas de um rio temporário no semiárido nordestino. Multitemas, 22(52): 53-66.

SANTOS, M. R. B., SALES, I. M. S., FEITOSA, F. D. S. A., FERREIRA, P. M. P., SOUSA, J. M. C., PERON, A. P., DEUS, M. S. M. 2015. Avaliação da biomassa de macrófias aquáticas presentes no rio guariba, Picos-PI. UFSCar, São Carlos, Reunião 
anual da Sociedade Brasileira para o Progresso da Ciência, 12 a 18 de julho.

SANTOS, V. R. \& LOLIS, S. F. 2012. Crescimento e competição por nutriente de macrófitas aquáticas flutuantes $S$. auriculata Aubl. e P. stratiotes L.. Seminário de Iniciação Científica - UFT, 11 a 14 de dezembro de 2012 - Campus de Palmas.

SILVA, E. C. V. \& FONTES, K. A. A. 2018. Macrófitas aquáticas no nordeste maranhense: levantamento florístico e chave de identificação. Bol. Mus. Para. Emílio Goeldi. Cienc. Nat., Belém, 13(3): 355-365.

SOOD, A., UNIYAL, P. L., PRASANNA, R., AHLUWALIA, A. S. 2012. Phytoremediation Potential of Aquatic Macrophyte, Azolla. AMBIO, 41: 122-137.

SOUSA, F. W. A. \& LIMA, I. M. M. F. 2019. Degradação ambiental do Rio Guaribas no trecho urbano do município de Picos-PI. XVIII Simpósio Brasileiro de Geografia Física Aplicada, Universidade Federal do Ceará, Fortaleza - CE, 11 a 15 de junho de 2019.

SOUZA, D. C., CUNHA, E. R., MURILLO, R. A., SILVEIRA, M. J., PULZATTO, M. M., DAINEZFILHO, M. S., LOLIS, L. A., THOMAZ, S. M. 2017. Species inventory of aquatic macrophytes in the last undammed stretch of the Upper Paraná River, Brazil.
Acta Limnologica Brasiliensia, 29(e115).

SOUZA, V. C. \& LORENZI, H. 2005. Botânica sistemática: guia ilustrado para identificação das famílias de Angiospermas da flora brasileira, baseada em APG II. Nova Odessa, SP: Instituto Plantarum.

THOMAZ, S. M. \& BINI, L. M. 1998. Ecologia e manejo de macrófitas aquáticas em reservatórios. Acta Limnologia Brasiliensia, 10(1): 103-116.

TORRES, C. R. M., FERNANDO, E. M. P., LUCENA, M. F. A. 2016. Checklist de plantas aquáticas em trechos de caatinga do semiárido paraibano, nordeste do Brasil. Gaia Scientia, 10(4): 284-296.

TRINDADE, R. R. T., PEREIRA, S.A.,ALBERTONI, E. F., PALMA-SILVA, C. 2010. Caracterização e importância das macrófitas aquáticas com ênfase nos ambientes límnicos do Campus Carreiros - FURG, Rio Grande, RS. Cadernos de Ecologia Aquática, 5 (2): 1-22.

WARD, J. V. 1998. Riverine landscapes: biodiversity patterns, disturbance regimes, and aquatic conservation. Biological Conservation, 83(3): 269-278.

YUAN, L. Y., LI, W., LIU, G. H. A., DENG, G. 2012. Effects of different shaded conditions and water depths on the growth and reproductive strategy of Vallisneria Spinulosa. Pakistan Journal of Botany, 44(3): 911. 